\title{
TRANSMISSION RANGE BASED OPTIMAL BACKBONE PATH FOR ENERGY CONSUMPTION IN MANET
}

\author{
Karmel A \\ Associate Professor Grade - I, School of Computer Science and Engineering, \\ Vellore Institute of Technology, Chennai, 600 127, Tamilnadu, India \\ karmel.a@vit.ac.in \\ Umamaheswari E \\ Associate Professor Grade - II, Center Faculty - Cyber Physical Systems, \\ Vellore Institute of Technology, Chennai, 600 127, Tamilnadu, India \\ umamaheswari.e@vit.ac.in \\ Kanchana Devi V \\ Associate Professor Grade - I, School of Computer Science and Engineering, \\ Vellore Institute of Technology, Chennai, 600 127, Tamilnadu, India \\ kanchanadevi@vit.ac.in \\ Kanimozhi G \\ Associate Professor Grade - I, School of Electrical Engineering, \\ Vellore Institute of Technology, Chennai, 600 127, Tamilnadu, India \\ kanimozhi.g@vit.ac.in \\ David Maxim Gururaj A \\ Associate Professor, Mathematics Division, School of Advanced Sciences, \\ Vellore Institute of Technology, Chennai, 600 127, Tamilnadu, India \\ davidmaxim.gururaj@vit.ac.in \\ Subhashini R \\ Associate Professor, Department of Information Technology, \\ Meenakshi College of Engineering, Chennai, Tamilnadu, India \\ drsubhashini05@gmail.com
}

\begin{abstract}
Mobile ad hoc network (MANET) is a decentralized, infrastructure less network, where the mobile nodes can transmit the information energetically. Optimizing the energy consumed during transmission is one among the essential requirements in MANET because of the restricted battery power and intricacy in replacing the battery. Transmission Range based Optimal Backbone Path (TROBP) algorithm is proposed to resolve this issue. TROBP algorithm constructs an optimal backbone path based on multi-criterion approach to minimize power consumption during transmission mode. Multi-Criterion approach is designed based on the periodic estimation of criteria's that includes transmission range and residual energy of optimal node, link stability of optimal backbone path and also the load distribution during transmission. TROBP algorithm implements a scheduling scheme for optimizing energy consumption in a high mobility environment.
\end{abstract}

Keywords: Backbone path; Link Stability; MANET; Multi-Criterion Approach; Power Consumption; Residual Energy; Transmission Range.

\section{Introduction}

Mobile Ad Hoc Network [MANET] is an assortment of versatile hubs that can be progressively set up with no fixed foundation. It is a self-governing framework where the versatile hosts associated by remote connections are allowed to move haphazardly and frequently act simultaneously. The geography of such organization is likely profoundly powerful in light of the fact that each organization hub can unreservedly move and no preintroduced base station exist. Because of the restricted remote transmission scope of every hub, information bundles might be sent along multi-bounces. Course development must be finished with at least overhead and 
data transmission utilization. Energy Efficient Routing Protocols [1] are trying to plan as execution corrupts because of versatility of portable specially appointed organization which is an open test.

This paper is coordinated as follows: The accompanying segment presents related works in improving energy utilization in MANET. Section 3 presents the TROBP calculation. Section 4 presents the numerical outcome. Area $\mathrm{V}$ closes the paper dependent on the execution done.

\section{Related Work}

Maher Heni et al. [2] actualized an upgraded rendition of AODV named PC-AODV (Power Control Ad hoc On Demand Distance Vector) where the occasional age of HELLO messages is limited to improve the energy utilization in MANET. Relative examination of the exhibition of AODV and PC-AODV is finished utilizing the measurements like ratio of packet delivery, overhead, Resultant Energy (RE) utilizing the test systems JIST/SWANS. The work is extended as assessing the PC-AODV exhibitions in various geographies and various kinds of portability to show the power of PC-AODV Protocol.

Kumar, N., et al [3] made a total report on power proficient directing conventions and on power sparing calculations for Mobile Ad Hoc Networks. Creators additionally recommended a Power Aware Techniques that consolidates the highlights of existing strategies like LEAR and GFR to diminish power utilization and to expand the organization life time. Energy-Aware Geo-area helped Routing (EAGER) [4] was a versatile steering plan for energy proficiency for enormous scope Ad Hoc Networks. Energetic joins proactive and receptive techniques. Excited first partitions the organization into intra-cell proactive directing and between cell responsive steering. Energy examination of EAGER is finished utilizing the components least transmission reach and number of bounces. The future improvements of EAGER is extended as: First, utilizing geographic sending rather than favorable to dynamic steering for intra-cell traffic; Second, actualizing between cell receptive traffic directing on a triangle/square organization segments rather on a hexagonal organization parcel for guaranteeing more proficient course disclosure; Third, multipath and acknowledgement directing into EAGER plan to adjust energy productivity and strength to bombed correspondences.

Sunho Lim [5] proposes a RandomCast correspondence system, to diminish the degree of catching by empowering the sender to start the favored catching level to accomplish a harmony among energy and steering execution. Creators tended to Route Discovery and support, Effect of catching, Unconditional catching and flat steering issue, Unconditional catching, Unconditional Rebroadcast and Semantic Discrepancy in DSR. Catching/rebroadcasting choice variables are neighbors count, Sender ID, energy consumed and portability. RandomCast conspire is executed utilizing number of neighbors. RandomCast plan can be improved by actualizing the other three elements: sender ID, Mobility and remaining battery energy and furthermore RandomCast can be incorporated with other directing conventions for better energy enhancement. Minimizing the complete force transmission and expanding the organization life time. The Authors actualized energy proficient calculations like DIStributed POWer "DISPOW", an advancing methodology that limits the transmission intensity of hubs. DISPOW utilizes multi-boundaries like availability, impedance and energy utilization to give better network and to adaptively oversee hub power. Creators additionally recorded calculations like MDR (Minimum Drain Rate), Multicast tree technique and Distributed Energy-Efficient specially appointed directing (DEAR) that amplifies the nodal battery life time, limits the postpone time and expands the organization life time.

Suganya, S. et al [7] proposed a methodology where an edge esteem is doled out on singular hub and all the bundles are sent with equivalent length for limiting the force utilization. The edge esteem is utilized by the relating hub to settle on a directing choice for parcel. During every transmission the energy level of every hub is checked against edge esteem. In the event that the energy level is lesser than the edge esteem, at that point a backup way to go will be resolved progressively to course the parcel.

Humaira Nishat et al [8] presented an Energy Efficient Dynamic Route Discovery (EEDRD) convention where the introduction of course revelation is done simply in the wake of sending some ideal number of information parcels dependent on the size and energy levels of hubs in the organization. EEDRD displays better execution contrasted with DRD by expanding the organization life expectancy as far as throughput, delay, bundle conveyance proportion and steering overhead.

Sivasnakar et al [9] proposed a two adjusted type of DSR calculation, for example, Cluster Based Energy Efficient Routing (CBEER) calculation and Energy Dynamic Source Routing-Route Update Cache (EEDSRRUC) calculation. Shirking of complete channel of energy structure a hub and the foundation of proficient energy mindful course is accomplished utilizing CBEER convention. EEDSR-RUC convention is planned so that choice with respect to the refreshing of course reserve will be done dependent on the energy level in the hub battery. These two conventions' exhibition is effective contrasted with existing DSR convention regarding ratio of packet delivery, average energy consumped and routing overhead. Pinki Nayak et al [10] talked about the different energy mindful directing conventions and the measurements answerable for energy advancements. The 
utilization of energy can be limited utilizing the accompanying methodologies: Transmission Power, Load Distribution and Sleep/Power-Down Mode Approaches.

\section{TROBP}

\subsection{Multi-Criterion Approach}

TROBP algorithm is designed based on a Multi-Criterion approach in which the following criteria's are involved.

Criteria1: The Source node selects the set of neighbor nodes that falls within its transmission range.

Criteria2: Source node selects one of the neighbor nodes whose residual energy is less that the Threshold value as the optimal node.

Criteria3: Packet must be transmitted only after slicing it into equal length.

Criteria4: Periodic evaluation of Link stability of optimal backbone path.

Criteria5: Periodic evaluation of transmission range and residual energy of optimal node.

Table 1: TROBP Algorithm

\begin{tabular}{|c|}
\hline Transmission Range baSed Optimal BaCKbONe Path Algorithm \\
\hline Source node: SN \\
\hline Destination node: DN \\
\hline Neighbor node: $\alpha_{1}, \alpha_{2}, \alpha_{3} \ldots \ldots \alpha_{n}$ \\
\hline Residual Energy: RE \\
\hline Threshold Limit Value: TLV \\
\hline Periodical Time Interval: $\mathrm{t}$ \\
\hline Selection of Optimal Path from SN to DN : \\
\hline Selection of optimal neighbour node: \\
\hline$\beta \leftarrow \mathrm{SN}$ \\
\hline Selection of neighbor node from $\beta_{j} \forall j=1$ to $m$ subject to constraint (1): \\
\hline For each $\alpha_{i} \forall i=1$ to $n$ \\
\hline Compute $R E\left(\alpha_{i}\right) \leftarrow E I\left(\alpha_{i}\right)-E S\left(\alpha_{i}\right)$ \\
\hline Select node with highest RE such that $R E\left(\alpha_{i}\right)>T L V$ \\
\hline Iterate until constraint (1) is obtained. \\
\hline If $R E\left(\alpha_{i}\right)>T L V$, transmission of data packets is done through these optimal nodes. \\
\hline The optimal backbone path $\phi \leftarrow\left\{S N, \beta_{\mathrm{j}} \forall j=1\right.$ to $\left.m, D N\right\}$ \\
\hline
\end{tabular}

\subsection{Determining Optimal Path through Optimal Node}

Mobile Ad hoc Network can be modeled as a bi-directed graph $B G=\langle N, L\rangle$ with set of nodes $\langle N\rangle$ and links $L(i, j) \forall i, j \in\langle N\rangle$, an optimal-backbone-path $\in$ NP composed of set of nodes and links subject to the constraint,

$$
\phi=\left\{\begin{array}{l}
\left\langle S N, \beta_{j}, D N\right\rangle \in\langle N\rangle \mid D\left(k, \beta_{j}\right)<T R_{k}, \forall j=1 \text { to } m \\
\text { and } R E\left(\beta_{j}\right)>T L V
\end{array}\right\}
$$

Neighbor node of a node $k$ is the set of all nodes that can be reached from node $\mathrm{k}$ subject to the constraint

$$
N B_{N_{k}}=D\left(k, \beta_{j}\right)<T R_{k} \forall j=1 \text { to } m
$$

The node $k$ selects the set of all nodes that lies within its transmission radius $T R_{k}$. Election of optimal node from the set of neighbor nodes $N B_{N_{k}}$ is based on the residual energy [11] subject to the constraint,

$$
R E\left(\beta_{j}\right)>T L V
$$


The neighbor node whose Residual energy which indicates the remaining energy of the node is greater than the Threshold Limit Value will be elected as optimal node. The entire process is repeated until an optimal path to the destination node is established. Since the optimal-backbone-path is in non-deterministic polynomial time class (Class NP), a polynomial time verifier algorithm that runs in polynomial time (Class P) is designed in table 1.

Table 2. Definitions for Notations

\begin{tabular}{|l|l|}
\hline Notations & Definitions \\
\hline$\langle N, L\rangle$ & Set of Nodes and Links in BG \\
\hline$\langle L\rangle$ & Set of Links \\
\hline SN,DN & Source Node, Destination Node \\
\hline$\alpha_{i}$ & Neighbor node \\
\hline$\beta_{j}$ & Optimal Neighbor node \\
\hline$R E\left(\alpha_{i}\right)$ & Residual Energy of node $\alpha_{i}$ \\
\hline$T L V$ & Threshold Limit Value \\
\hline$E I\left(\alpha_{i}\right)$ & Initial Energy level of Node $\alpha_{i}$ \\
\hline$E S\left(\alpha_{i}\right)$ & Current Energy level of node $\alpha_{i}$ \\
\hline$\phi$ & Optimal Backbone Path \\
\hline$L_{p a c}$ & Length of Packet \\
\hline$O L_{p a c}$ & Optimal Length of Packet \\
\hline LCF & Link Capacity Factor \\
\hline NBP & Number of Bits per packet \\
\hline LSR & Link Stability Record \\
\hline
\end{tabular}

\subsection{Link Stability}

If any one of the optimal node whose Residual energy falls less than the Threshold Limit Value or if any one of the optimal node that moves far behind transmission radius of the source node/transmitting node, then reestablishment of optimal backbone path must be done. The stability of the each link in the optimal-backbonepath is evaluated for every periodic interval $t$.

For all optimal nodes pairs in the optimal path set, find the corresponding link stability. Each optimal node maintains Link Stability Record. Link Stability must be periodically confirmed for all these set of pairs: $\{\mathrm{SN}$, Inter_Node $\},\{$ Inter.node, Inter.node $\},\{$ Inter.node, DN $\}$. Link Stability Depends on Factor $1 \& 2$ :

A. If the node in a pair lies within the transmission range of each other node then the link is stable.

B. If the packet is too long, link will be unstable frequently, that results in packet drop.

C. If the packet is too short, packet overhead occurs and throughput will be reduced.

D. Slicing of packet is necessary. Optimal packet length needs to be determined.

\section{Factor1:}

For every periodic time interval, each optimal node in the optimal backbone Path set should check whether its optimal neighbor node is within its communication range. If so the packet of length $L_{p a c}$ is transmitted. Optimal Backbone path gets updated periodically by running the algorithm in Table II.

\section{Factor2:}

Packet Slicing is meant only for long length packet. Packet should be sliced in such a way that the link stability must exists till end of transmission between end nodes. OLpac be the optimal length of each sliced packet which is based on the slicing criteria given below.

$$
N B P \leq L C F
$$

NBP denotes the number of bits per packet and Link capacity factor (LCF) which is associated with each optimal node pair denotes the maximum number of bits per packets that can be transmitted over the link. 


\subsection{Load Distribution Approach}

Packet slicing is done once an optimal path from source to destination is established. Packets are sliced into equal length packets and transferred along the optimal-backbone-path. If packets of unequal length are transmitted, the energy consumed by each optimal node may vary. This may lead to complete drain out of energy at least in any one of the optimal node and the optimal-backbone-path has to be re-established again for the transfer of entire packets. As a result, the network performance will automatically decrease. To avoid this criticality packet slicing is done in order to minimize the energy consumed during transmission of packets.

\section{RESULTS}

TROBP algorithm is implemented. First the algorithm is implemented without considering the link stability and packet slicing. Second the TROBP algorithm is done including the link stability and packet slicing schemes. The Mathematical results shows better results under energy consumption during transmission only when the link stability and packet slicing schemes are included. The total energy consumed per packet during transmission through the optimal nodes ${ }^{\beta_{j}}$ form source to destination subject to constraint (1) must be minimized. The energy consumed per packet is evaluated based on the following equation,

$$
\operatorname{Min} E=\sum_{j=0}^{D N-1} T\left(\beta_{j}, \beta_{j+1}\right)
$$

where, $\beta_{j}, \beta_{j+1}$ are optimal nodes that falls in optimal-backbone-path; $T$ denotes the energy consumed during transmitting of a packet over one hop.

Table 3. Simulation Parameters

\begin{tabular}{|l|l|}
\hline \multicolumn{1}{|c|}{ Parameters } & \multicolumn{1}{c|}{ Attributes } \\
\hline Simulator & NS-2.34 \\
\hline Routing Protocol & AODV \\
\hline Simulation Duration & $200 \mathrm{sec}$ \\
\hline Number of nodes & 50 \\
\hline Transmission Range & $250 \mathrm{~m}$ \\
\hline Movement Model & Random Waypoint \\
\hline Antenna & Omni Antenna \\
\hline Radio Propagation & Two Ray Propagation \\
\hline MAC Layer Protocol & IEEE 802.11 \\
\hline Maximum Speed & $20 \mathrm{~m} / \mathrm{sec}$ \\
\hline Traffic Type & CBR \\
\hline Payload & 512 bytes $/$ packet \\
\hline Pause Time & $100 \mathrm{sec}$ \\
\hline
\end{tabular}




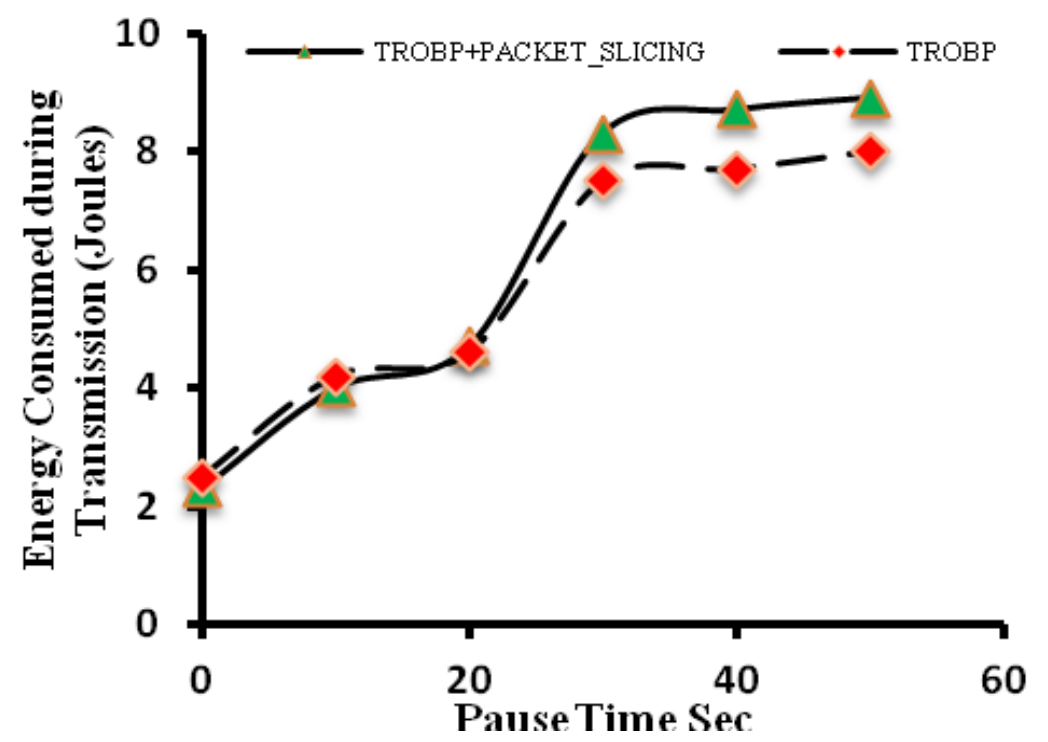

Fig. 1. Energy Consumed During Transmission

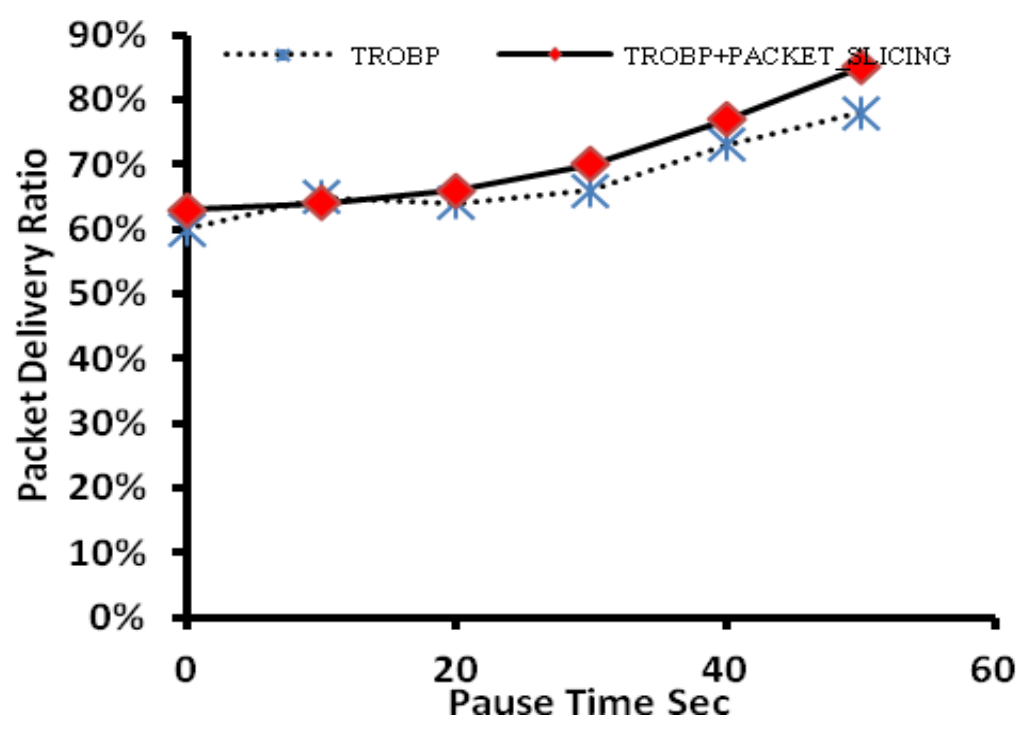

Fig. 2. Packet Delivery Ratio

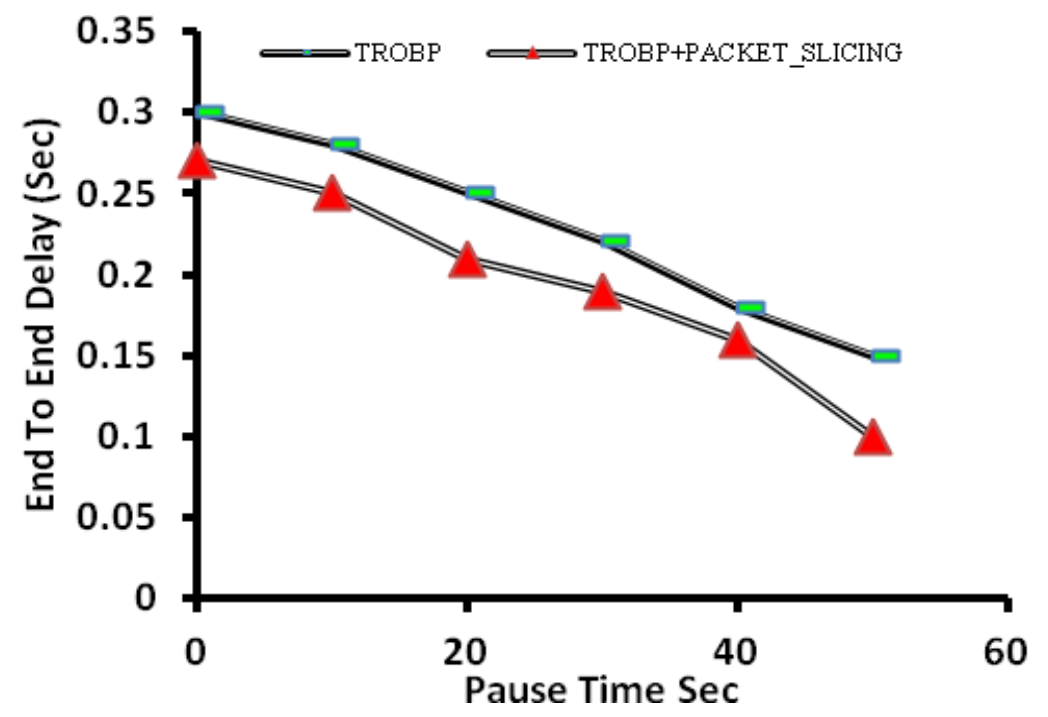

Fig. 3. End to End Delay 


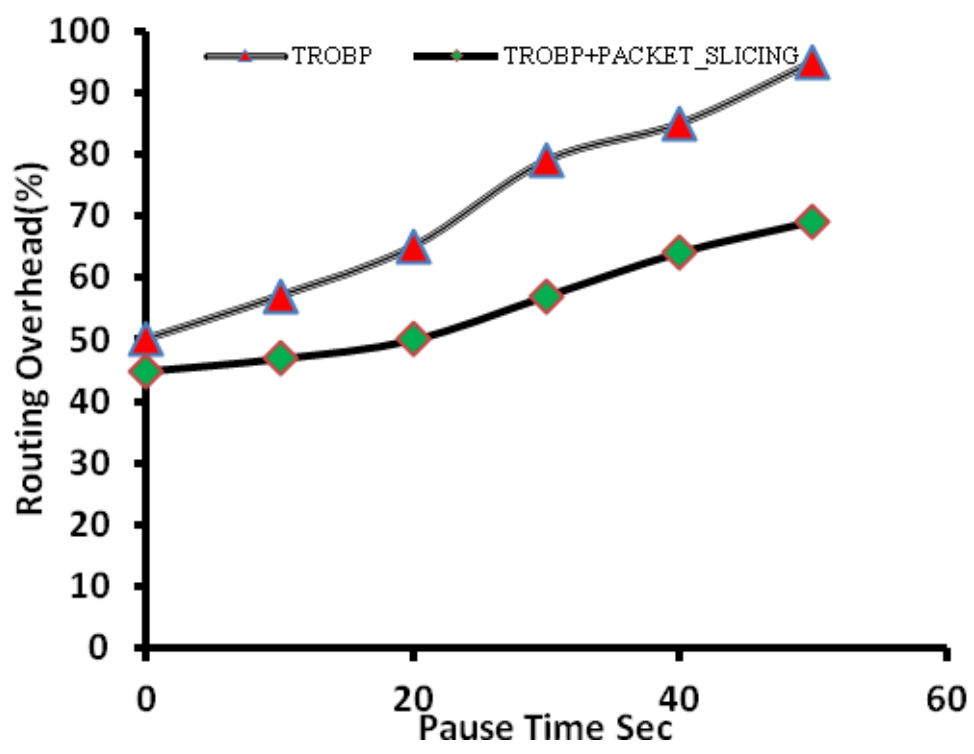

Fig. 4. Routing Overhead

Fig.1 shows the simulations results for the Energy Consumed during transmission of packets. Initially TROBP algorithm is implemented and packet transmission is done without packet slicing i.e., packets are transmitted without any segmentation. The same TROBP algorithm is implemented and data transmission is done by slicing the packets into equal length. Energy is measured in Joules. The simulation results shows that energy consumed during transmission with packet slicing give better results.

Fig. 2 shows the simulation results of the number of packets delivered. The simulation results sows that the number of packets with packet slicing give better results. Around some $10 \%$ increase in the number of packets delivered through packet slicing technique.

Fig. 3 \& Fig. 4 shows the simulation results of the end-to-end delay and Routing overhead respectively. The simulation results show that both end-to-end delay and routing overhead are minimum, when TROBP algorithm is implemented with packet slicing.

\section{Conclusion}

The TROBP algorithm is designed for evaluating energy consumption during transmission of packets. The Simulation results shows better results when TROBP algorithm is implemented with packet slicing technique. The algorithm also projects the necessity of link stability and equal load distribution during transmission of packets.

\section{References}

[1] Jinhua Zhu and Xin Wang, "Model and Protocol for Energy-Efficient Routing over Mobile Ad Hoc Networks", IEEE Transaction on Mobile Computing, Vol.10, No.11, pp1546 - 1557, 2011.

[2] Maher Heni and Ridha Bouallegue, "Power Control in Reactive Routing Protocol for Mobile Ad Hoc Network", International Journal of Wireless and Mobile Networks, Vol. 4, No. 2, 2012

[3] Kumar, N., Suresh Gnana Dhass, C, "A Complete Study on Energy Efficient Techniques for Mobile Adhoc Networks", International Journal of Advanced Research in Computer Science and Software Engineering, Vol. 2, No.9, pp.129-133, 2012.

[4] Qing Zhao, Lang Tong, and David Counsil, "Energy-Aware Adaptive Routing for Large-Scale Ad Hoc Networks: Protocol and Performance Analysis", IEEE Transactions on Mobile Computing, Vol. 6, No. 9 , pp.1048-1059, 2007.

[5] Sunho Lim, Chansu Yu and Chita Das, R. "RandomCast: An Energy-Efficient Communication Scheme for Mobile Ad Hoc Networks", IEEE Transactions on Mobile Computing, Vol. 8, No. 8, pp.1039-1051, 2009.

[6] Anjum Asma, Gihan Nagib, "Energy Efficient Routing Algorithms for Mobile Ad Hoc Networks -A Survey", International Journal of Emerging Trends and Technology in Computer science. Vol. 1 No. 3 (2012) 218-223

[7] Suganya S., Palaniammal S., "A Dynamic Approach to Optimize Energy Consumption in Mobile Adhoc Network", European Journal of Scientific Research. Vol. 85 No. 2 (2012) 225-232

[8] Humaira Nishat, Sreenivasa Rao, D.," Energy Efficient Dynamic Rute Discovery Protocol for Mobile Ad Hoc Networks", International Journal of Computer Applications, Vol. 56,No. 7, pp.44-47, 2012.

[9] Sivasankar, p., Chellapan, C., Balaji, S., "Optimized Energy Efficient Routing Protocols and their Performance Comparison for MANET", European Journal of Scientific Research, Vol. 9, No. 1, 136-148, 2012.

[10] Pinki Nayak, Rekha Agarwal, Seema Verma, "An Overview of Energy Efficient Routing Protocols in Mobile Ad Hoc Network", International Journal of Research and Reviews in Ad hoc Networks, Vol. 2, No. 1 93-96, 2012.

[11] Suganya.S, Palaniammal.S, “An Optimized Energy Consumption Algorithm in MANET”, Procedia Engineering 38, pp.903-910, 2012.

[12] F. Guerriero *, F. De Rango, S. Marano, E. Bruno. "A biobjective optimization model for routing in mobile ad hoc networks", Applied Mathematical Modelling 33, pp.1493-1512, 2009.

[13] Jayakumar C and Chellappan C ,"Quality of Service in Associativity based Mobility-Adaptive K-Clustering in Mobile Ad-hoc Networks", International Journal of The Computer, the Internet and Management, Vol.14, No.1, pp.61-80, 2006. 
[14] Nityananda Sarma and Sukumar Nandi, "A Multipath QoS Routing with Route Stability for Mobile Ad Hoc Networks", IETE Technical Review, Vol.27, No.5, pp.380-397, 2010

[15] Jayakumar C and Chellappan C, "Optimized on demand routing protocol of mobile adhoc network", Informatica, Vol.17, No. 4, pp.481-502, 2006.

[16] P. Infant Kingsly, C. Jayakumar, Mahendran Sadhasivam, S. Deepan Chakravarthy, "Smart Way for Secured Communication in Mobile Ad-hoc Networks", International Journal Computational Intelligence and Informatics, Vol.2 No. 1, pp.1-9, 2012.

[17] Karmel A and Dr.C.Jayakumar, "Analysis of MANET Routing Protocols Based on Traffic Type", International Journal of Research in Engineering \& Advanced Technology (IJREAT), Vol.1, Issue 1, pp.1-4, 2013.

[18] Jayakumar C and Chellappan C, "A QoS aware energy efficient routing protocol for wireless -ad-hoc networks", Asian Journal of Information Technology, Vol.4, No.6, pp.578-582, 2005.

[19] Satoshi Yamazaki, Yu Abiko, Hideki Mizuno, "A Simple and Energy-Efficient Flooding Scheme for Wireless Routing", Wireless communications nad MObile Computing [Special Issue: Recent Advances in Green Communications and Networks, Vol. 2020, Article ID 8832602 ,.https://doi.org/10.1155/2020/8832602 